\title{
Spectrally Selective Imaging with Wideband Balanced Steady-State Free Precession MRI
}

\author{
Tolga Çukur ${ }^{1,2 *}$
}

Purpose: Unwanted, bright fat signals in balanced steadystate free precession sequences are commonly suppressed using spectral shaping. Here, a new spectral-shaping method is proposed to significantly improve the uniformity of stopband suppression without compromising the level of passband signals.

Methods: The proposed method combines binomial-pattern excitation pulses with a wideband balanced steady-state free precession sequence kernel. It thereby increases the frequency separation between the centers of pass and stopbands by $\pi$ radians, enabling improved water-fat contrast. Simulations were performed to find the optimal flip angles and subpulse spacing for the binomial pulses that maximize contrast and signal efficiency.

Results: Comparisons with a conventional binomial balanced steady-state free precession sequence were performed in simulations as well as phantom and in vivo experiments at $1.5 \mathrm{~T}$ and $3 \mathrm{~T}$. Enhanced fat suppression is demonstrated in vivo with an average improvement of $58 \%$ in blood-fat and $68 \%$ in muscle-fat contrast ( $P<0.001$, Wilcoxon signed-rank test).

Conclusion: The proposed binomial wideband balanced steady-state free precession method is a promising candidate for spectrally selective imaging with enhanced reliability against field inhomogeneities. Magn Reson Med 75:11321141, 2016. (c) 2015 Wiley Periodicals, Inc.

Key words: steady-state free precession; spectral; selectivity; RF pulse; excitation; fat suppression; wideband including angiography (4-7), musculoskeletal imaging $(8,9)$, cellular imaging $(10,11)$, interventional imaging $(12,13)$, and parameter mapping (14-16). At the same time, however, bSSFP sequences yield a $\mathrm{T}_{2} / \mathrm{T}_{1}$-weighted contrast that depicts fat brighter than tissues of primary interest such as blood or muscle, degrading image quality. Therefore, reliable suppression of fat signal is critical for acquiring high quality bSSFP images.

Many sophisticated approaches have been previously proposed for either fat suppression or fat-water separation in bSSFP imaging (17-29). These approaches differ in the way that they introduce the spectral selectivity required to disentangle fat and water signals. Phasesensitive techniques commonly require the acquisition of multiple bSSFP images and subsequent postprocessing to estimate the fat-water distribution in bSSFP images (17-21). While these techniques generally achieve robust fat/water separation across large volumes, they incur substantially prolonged scan times and susceptibility to partial volume effects. Another group of methods reduces fat signal by using specific excitation trains during the transient phase of bSSFP sequences (22-24). These transient-suppression techniques yield relatively shorter scan times, but signal instabilities and magnetization decay during the transient phase can lead to image artifacts (30).

Alternatively, the bSSFP spectral profile can be reshaped through periodic manipulation of radiofrequency (RF) excitations and repetition times (TR) (25-29). Spectral-shaping techniques achieve selective imaging by creating a passband near the water resonance and a stopband near the fat resonance. Because these techniques rely on steady-state magnetization profiles, they can alleviate problems due to partial volume effects and transient signal behavior. However, suppression levels are generally nonuniform across the stopband, and fat suppression can deteriorate with moderate to large B0 field inhomogeneity $(25,28)$.

In this work, we propose a new spectral-shaping strategy for selective bSSFP imaging with improved robustness against B0 field inhomogeneities. The proposed method combines spectrally selective binomial RF excitations with a wideband bSSFP kernel (31,32). This approach provides a more favorable trade-off between passband efficiency and stopband suppression when compared to the conventional use of spectral RF excitations in bSSFP sequences $(27,33,34)$. Thus it enables the use of higher-order binomial excitation patterns for enhanced fat suppression while maintaining high passband signal levels. We present simulations, phantom and in vivo data that demonstrate the performance improvements attained using the proposed spectral-shaping method.
${ }^{1}$ Department of Electrical and Electronics Engineering, Bilkent University, ${ }^{2}$ National Magnetic Resonance Research Center, Bilkent University, Ankara, Turkey.

Grant sponsor: TUBITAK 2232 Fellowship; Grant number: 113C011; Grant sponsor: Marie Curie Actions Career Integration Grant; Grant number: Organization Installation Grant; Grant number: IG 3028.

${ }^{*}$ Correspondence to: Tolga Çukur, Ph.D.; Department of Electrical and Electronics Engineering, Room 304, Bilkent University, Ankara, TR-06800, Turkey. E-mail: cukur@ee.bilkent.edu.tr

Received 10 October 2014; revised 26 January 2015; accepted 25 February 2015

DOI $10.1002 / \mathrm{mrm} .25700$

Published online 4 April 2015 in Wiley Online Library (wileyonlinelibrary.com). 


\section{METHODS}

\section{Spectral Shaping of bSSFP Profiles}

Regular bSSFP sequences have a frequency-dependent magnetization profile $(3,35)$ :

$$
M\left(\alpha, \Delta \phi, \phi_{\mathrm{RF}}\right)=\left|M_{\mathrm{ss}} \frac{1-A e^{-i\left(\Delta \phi+\phi_{\mathrm{RF}}\right)}}{1-B \cos \left(\Delta \phi+\phi_{\mathrm{RF}}\right)}\right|
$$

where $\alpha$ denotes the RF flip angle, $\Delta \phi=2 \pi \cdot \Delta f$. TR denotes the phase accrual per TR at an off-resonant frequency $\Delta f$, and $\phi_{\mathrm{RF}}$ denotes the RF phase increment across TRs. The remaining terms $M_{\mathrm{ss}}, A$, and $B$ depend on sequence and tissue parameters (see Appendix).

In conventional bSSFP imaging, $\phi_{\mathrm{RF}}=\pi$ is selected to center the passband at the on-resonant frequency $(\Delta \phi=0)$. Assuming $\mathrm{TR} \ll\left(\mathrm{T}_{1}, \mathrm{~T}_{2}\right)$, the resulting signal can then be approximated as (36) (see Appendix):

$$
M_{\mathrm{pass}}(\alpha) \approx \frac{M_{o} \sin \alpha}{\left(\frac{\mathrm{T}_{1}}{\mathrm{~T}_{2}}+1\right)-\left(\frac{\mathrm{T}_{1}}{\mathrm{~T}_{2}}-1\right) \cos \alpha}
$$

For moderate to large flip angles (e.g., $\alpha>10^{\circ}$ ), $M_{\text {pass }}(\alpha)$ yields a relatively high signal intensity and a $\mathrm{T}_{2} / \mathrm{T}_{1^{-}}$ weighted contrast. However, the signal intensity diminishes proportionally with $\alpha$ for smaller flip angles (e.g., $\alpha<5^{\circ}$ ):

$$
M_{\text {pass }}\left(\alpha<5^{\mathrm{o}}\right) \approx \frac{M_{\mathrm{o}}}{2} \alpha
$$

which is obtained using second-order Taylor series approximations for sine and cosine functions $(<1 \%$ approx. error).

As seen in Eqs. [2] and [3], spectral selectivity of the magnetization profile can be manipulated by generating a frequency-dependent RF flip angle, $\alpha(f)$. High flip angles should be maintained near the frequency band of the tissue of interest (e.g., water), whereas flip angles should be lowered in the frequency band of the contaminating tissue (e.g., fat). This can be achieved by designing an RF excitation pulse that generates the transverse magnetization $M_{\mathrm{RF}}(\Delta f)=M_{o} \sin (\alpha(\Delta f))$.

Binomial RF pulses are excellent candidates to generate a frequency-dependent flip angle due to their simple design, robustness against B1 field inhomogeneities, and approximately Gaussian spectral profiles with minimal ripples $(27,33,34)$.

Binomial subpulse amplitudes are proportional to binomial coefficients (e.g., "1 2 1" for third order), and subpulses are spaced at regular intervals $\tau$ (Fig. 1a). The resulting excitation profile is periodic with $f_{\mathrm{b}}=1 / \tau$, and the spacing between the null $\left(\alpha_{\text {null }}\right)$ and peak $\left(\alpha_{\max }\right)$ points of the profile is $f_{\mathrm{b}} / 2=1 / 2 \tau$. Ideally, $\tau$ should be selected to equate this spacing with the frequency separation between water and fat. In addition, subpulse phases should be selected to align $\alpha_{\text {null }}$ with the fat resonance. Assuming negligible relaxation effects $\left(\tau \ll \mathrm{T}_{1}, \mathrm{~T}_{2}\right)$, the flip angle generated by the binomial pulse equals zero when the phase accrual between consecutive subpulses (due to both off-resonance and subpulse phases) is $\pi$ radians.

Flip angle profiles and corresponding magnetization profiles for binomial pulses in a $\phi_{\mathrm{RF}}=\pi$ cycled bSSFP sequence are displayed in Figure 1a. A first-order binomial pulse "1" creates a uniform flip angle profile, and it is equivalent to a regular bSSFP sequence. Assuming a stopband centered at $\Delta \phi=0$ with no loss of generality, a second-order binomial pulse "1 -1 ," equivalently the fat-suppressing alternating-TR (FSATR) sequence (28), selectively produces near-zero flip angles around on-resonance. However, because the null region of the flip angle profile is narrow, the level of suppression across the generated stopband is nonuniform.

The null regions of the flip angle profile can be broadened by third and higher-order pulses as seen in Figure 1a. Considering that practical TR values for a binomial bSSFP sequence are around 4-5 ms (33), the passbands centered at $\Delta \phi=2 \pi$ and $\Delta \phi=4 \pi$ can be used to image water at $1.5 \mathrm{~T}$ and $3 \mathrm{~T}$, respectively. Unfortunately, higher-order pulses reduce the flip angles and cause signal degradation in the neighboring passbands particularly at lower field strengths (34). Although lengthening subpulse spacing $(\tau)$ may alleviate this degradation, it will lengthen TR and increase susceptibility to B0 field inhomogeneity.

\section{Spectral Shaping with Wideband bSSFP}

Here, we propose an alternative spectral-shaping strategy to enhance stopband uniformity without degrading the passband signal levels. We incorporate binomial pulses into a bSSFP sequence with $\phi_{\mathrm{RF}}=0$ phase cycling (as opposed to $\phi_{\mathrm{RF}}=\pi$ in FS-ATR). Figure $1 \mathrm{~b}$ displays the flip angle profiles and resulting bSSFP magnetization profiles generated by binomial pulses of orders 1-4. A second-order binomial pulse " $1-1$," equivalently the wideband bSSFP sequence (31), selectively yields nearzero flip angles around on-resonance. Note that while the flip angle profile of the " $1-1$ " pulse is identical for $\phi_{\mathrm{RF}}=0$ and $\phi_{\mathrm{RF}}=\pi$ bSSFP sequences, reduced flip angles near the bSSFP signal null do not yield effective stopband suppression (Fig. 1b).

To understand the effect of reduced flip angles near bSSFP signal nulls, we can revisit the approximations to the bSSFP signal equation (Eq. [1]). Taking $\Delta \phi=0, \phi_{\mathrm{RF}}$ $=0$ and $\mathrm{TR} \ll\left(\mathrm{T}_{1}, \mathrm{~T}_{2}\right)$, the magnetization level near $\mathrm{a}$ bSSFP null can be approximated as (see Appendix):

$$
M_{\mathrm{null}}(\alpha) \approx \frac{M_{o} \sin \alpha}{\left(\frac{2 \mathrm{~T}_{1}}{\mathrm{TR}}-\frac{\mathrm{T}_{1}}{\mathrm{~T}_{2}}-1+\frac{\mathrm{TR}}{\mathrm{T}_{2}}\right)-\left(\frac{2 \mathrm{~T}_{1}}{\mathrm{TR}}-\frac{\mathrm{T}_{1}}{\mathrm{~T}_{2}}-1\right) \cos \alpha}
$$

For $\alpha>10^{\circ}$, the $\mathrm{T}_{1} / \mathrm{TR}$ term in the denominator generates the well-known bSSFP null. However, for smaller flip angles, the magnetization can be approximated as:

$$
M_{\text {null }}\left(\alpha<5^{o}\right) \approx M_{\mathrm{o}} \frac{\alpha}{\left(\frac{\mathrm{TR}}{\mathrm{T}_{2}}\right)}
$$

Contrary to Eq. [3], given a sufficiently small $\mathrm{TR} / \mathrm{T}_{2}$ value, reasonably high signal levels can be generated even for flip angles around $\alpha=1^{\circ}$. Thus the flip angle should be lowered as much as possible to ensure reliable stopband suppression. One can assume that the " $1-1$ " binomial pulse readily creates a perfect $\alpha=0^{\circ}$ at $\Delta \phi=0$. 

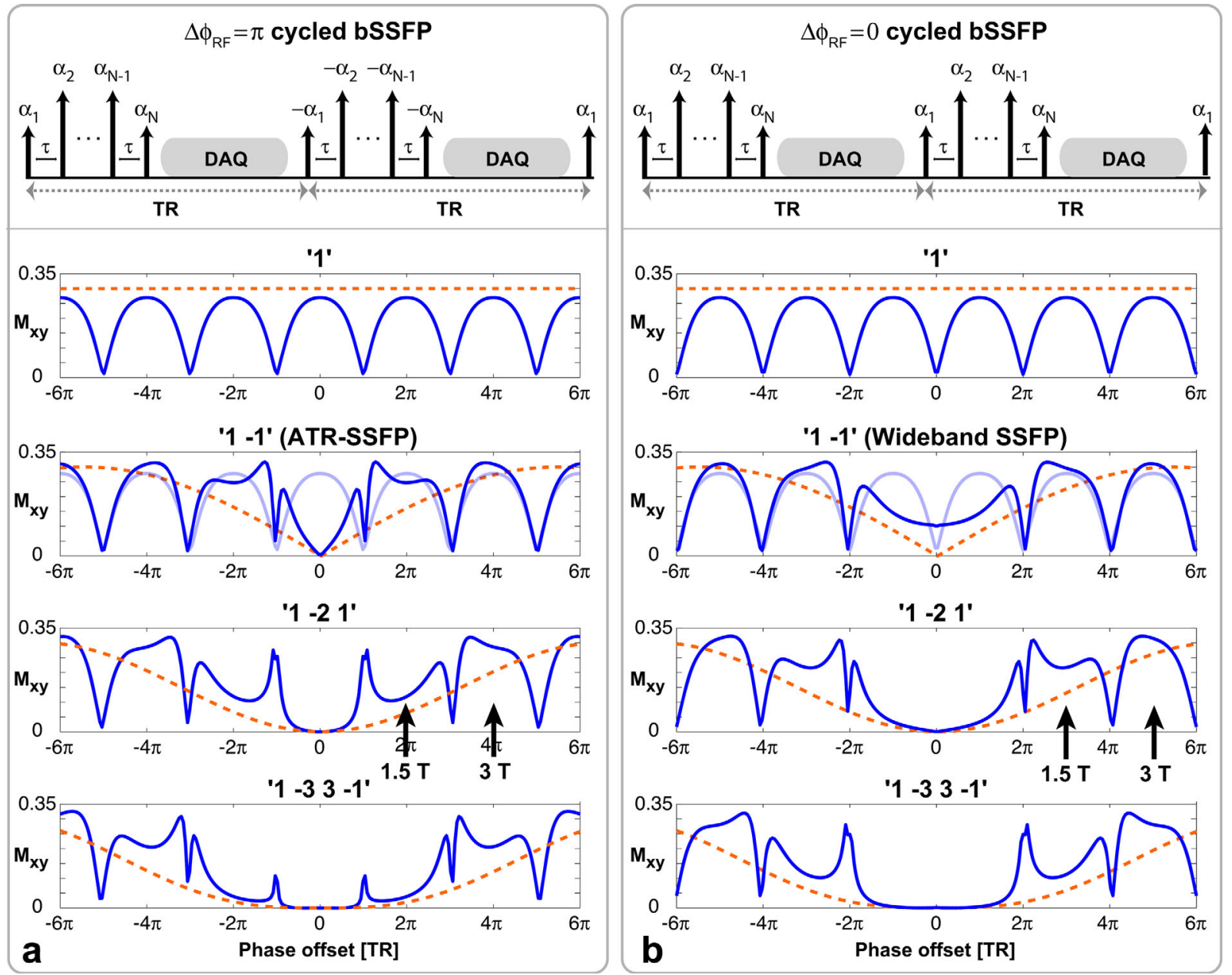

FIG. 1. a: Diagram for a binomial-pulse bSSFP sequence with $\phi_{\mathrm{RF}}=\pi$ RF phase cycling (top row). The binomial pulse contains subpulses of flip angles $\alpha_{1, \ldots, n}$ spaced at a regular interval of $\tau$, and it generates a frequency-dependent flip angle $\alpha(f)$. This in turn modifies the shape of the bSSFP magnetization profile (bottom rows). The frequency-dependent flip angle (dashed orange line) and the resulting profile (solid blue line) are shown for binomial pulses of orders 1 to 4 ("1," "1-1," "1 -2 1," and "1 $-33-1$ "). Flip angle and magnetization profiles are displayed as a function of the phase offset per TR $(\Delta \phi)$. Water-selective imaging can be performed using the passband centered at $\Delta \phi=2 \pi$ at $1.5 \mathrm{~T}$, and that centered at $\Delta \phi=4 \pi$ at $3 \mathrm{~T}$. Higher-order pulses broaden the null region of $\alpha(f)$, improving stopband suppression at the expense of reducing passband signals. b: Diagram for a binomial-pulse bSSFP sequence with $\phi_{\mathrm{RF}}=0 \mathrm{RF}$ phase cycling (top row). The resulting magnetization profiles are shown for binomial pulses of orders 1-4 (bottom rows). Water-selective imaging can be performed using the passband centered at $\Delta \phi=3 \pi$ at $1.5 \mathrm{~T}$, and that centered at $\Delta \phi=5 \pi$ at $3 \mathrm{~T}$. Due to increased spacing between pass and stopbands, higher-order pulses improve stopband suppression without compromising passband signals.

However, relaxation effects during the finite subpulse intervals $(\tau)$ lead to deviations from the target flip-angle profile, generating considerably high bSSFP signal levels (Fig. 1b).

To address this critical issue, we propose to improve flip angle profiles by using higher-order binomial pulses. Figure 1b clearly shows that third and fourth-order binomial pulses create highly uniform stopbands with enhanced suppression compared to binomial $\phi_{\mathrm{RF}}=\pi$ cycled bSSFP sequences. Note that the proposed method uses the passbands are centered at $\Delta \phi=3 \pi$ and $\Delta \phi=5 \pi$ for water imaging at $1.5 \mathrm{~T}$ and $3 \mathrm{~T}$, respectively. The separation between stop and passbands is increased by $\pi$ radians compared to FS-ATR, and as a result, the proposed method also alleviates signal degradation in the neighboring passbands.

\section{Effect of Subpulse Spacing on bSSFP Profiles}

Given a resonant-frequency difference of 3.5 ppm between fat and water, the ideal subpulse spacing is $\tau$ $=1.15 \mathrm{~ms}$ at $1.5 \mathrm{~T}$ and $\tau=0.58 \mathrm{~ms}$ at $3 \mathrm{~T}$. However, the total TR in a binomial pulse bSSFP sequence is $\mathrm{TR}=\mathrm{TR}_{l}+(n-1) \tau$, where $\mathrm{TR}_{l}$ is the data acquisition interval between consecutive pulses, and $n$ is the pulse order. Because relatively long $\tau$-values lengthen the overall TR, it is desirable to understand the effects of more practical $\tau$ values on the magnetization profiles.

For this purpose, we simulated the steady-state magnetization profiles of two sample sequences: a " $1-1$ " $\phi_{\mathrm{RF}}=\pi$ bSSFP sequence and a " $1-21$ ” $\phi_{\mathrm{RF}}=0 \mathrm{bSSFP}$ sequence. Simulations were performed for $\mathrm{T}_{1} / \mathrm{T}_{2}=1200 /$ $250 \mathrm{~ms}, \mathrm{TR}_{I}=4.0 \mathrm{~ms}$, and $\tau \in\left[\begin{array}{ll}0.3 & 1.0\end{array}\right] \mathrm{ms}$. For both sequences, the flip angles for the binomial subpulses 

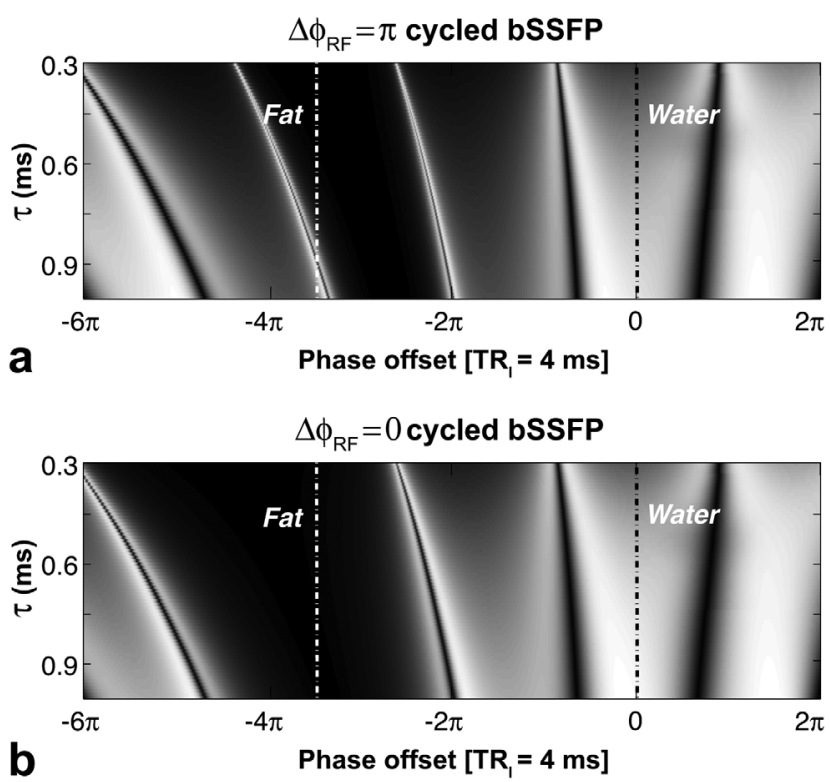

FIG. 2. a: Simulated magnetization profile of a $\phi_{\mathrm{RF}}=\pi$ bSSFP sequence with a "1-1" binomial pulse, is shown as a function of the phase offset per $\mathrm{TR}_{l}=4 \mathrm{~ms}$. The location of water and fat resonances are marked with dashed black and white lines, respectively. b: Magnetization profile of a $\phi_{\mathrm{RF}}=0$ bSSFP sequence with a "1 -21 " binomial pulse. Simulations for both sequences were performed using $\alpha_{\max }=80^{\circ}$ for the binomial

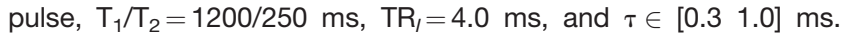
For both sequences, longer $\tau$ values lengthen the overall TR and increase susceptibility to BO field inhomogeneities. At the same time, longer $\tau$ increases the flip angle created by the binomial pulse at the water passband, improving passband signal levels.

were set to yield a maximum flip angle of $\alpha_{\max }=80^{\circ}$ (e.g., $20^{\circ}-\left(-40^{\circ}\right)-20^{\circ}$ for the “ $1-21$ ” pulse). The spectral profiles were shifted across the frequency axis to align the center of the passband with the water resonance $(\Delta \phi=0)$ at $3 \mathrm{~T}$.

The simulated profiles in Figure 2 suggest that shorter $\tau$ values that yield shorter TR broaden the bSSFP pass and stopbands, improving fidelity against B0 field inhomogeneities. At the same time, however, shorter $\tau$ values also broaden the binomial excitation profile. Given the theoretical constraints on $\tau$ for fat-water separation $(\tau=$ $1.15 \mathrm{~ms}$ at $1.5 \mathrm{~T}$ and $\tau=0.58 \mathrm{~ms}$ at $3 \mathrm{~T}$ ), this broadening will either reduce the passband signal or yield suboptimal stopband suppression.

\section{Optimization of Sequence Parameters}

The trade-off between stopband suppression and passband efficiency is biased strongly by the flip angle and subpulse spacing. In practice, the optimal $\tau$ that maximizes the expected water-fat contrast over bSSFP bands will critically depend on the prescribed $\alpha_{\max }$ value. To determine optimal sequence parameters, we simulated the magnetization profiles of water and fat separately for binomial bSSFP sequences with $\phi_{\mathrm{RF}}=\pi$ and $\phi_{\mathrm{RF}}=0$. Simulations were performed with the following parameters: $\mathrm{T}_{1} / \mathrm{T}_{2}=1200 / 250 \mathrm{~ms}$ for water, $\mathrm{T}_{1} / \mathrm{T}_{2}=270 / 85 \mathrm{~ms}$ for fat (37), $\mathrm{TR}_{l}=4.0 \mathrm{~ms}, \tau \in\left[\begin{array}{ll}0.3 & 1.0\end{array}\right] \mathrm{ms}, \alpha_{\max } \in\left[30^{\circ}\right.$ $120^{\circ}$, and binomial pulse order of 2-4. Magnetization profiles were shifted to center the passbands on the water resonance.

For each sequence, the level of stopband suppression was first quantified as the difference between average water and fat signals across a $[-100100] \mathrm{Hz}$ frequency range (i.e., water-fat contrast). Second, the efficiency of the passband was quantified as the average water signal normalized by the square root of the total TR (SNR efficiency). Finally, an aggregate performance metric was derived for each sequence by multiplying the contrast and SNR efficiency factors.

The aggregate performance metric for the conventional and proposed sequences at $1.5 \mathrm{~T}$ and $3 \mathrm{~T}$ are displayed in Figures 3 and 4 . The " $1-1$ " binomial pulse yields the best performance among $\phi_{\mathrm{RF}}=\pi$ bSSFP sequences. Near-optimal sequence parameters at $1.5 \mathrm{~T}$ are $\alpha_{\max } \in$ $\left[\begin{array}{ll}60^{\circ} & 90^{\circ}\end{array}\right]$ and $\tau \in\left[\begin{array}{ll}0.6 & 0.9\end{array}\right] \mathrm{ms}$, whereas at $3 \mathrm{~T}$ they are $\alpha_{\max } \in\left[\begin{array}{ll}40^{\circ} 70^{\circ}\end{array}\right]$ and $\tau \in\left[\begin{array}{ll}0.4 & 0.6\end{array}\right] \mathrm{ms}$. In comparison, the "1 -21 " binomial pulse achieves the best performance for the proposed sequence. In this case, near-optimal parameters at $1.5 \mathrm{~T}$ are $\alpha_{\max } \in\left[\begin{array}{ll}40^{\circ} & 80^{\circ}\end{array}\right]$ and $\tau \in\left[\begin{array}{ll}0.8 & 1.0\end{array}\right]$ $\mathrm{ms}$, whereas at $3 \mathrm{~T}$ they are $\alpha_{\max } \in\left[40^{\circ} 70^{\circ}\right]$ and $\tau \in[0.5$ 0.8 ms.

\section{Transient Behavior of Magnetization}

Magnetization levels of bSSFP sequences exhibit wellknown oscillations during the transient phase before steady state is reached $(30,36)$. Strongest oscillations usually occur near the bSSFP nulls with zero steadystate magnetization (30). Bloch simulations were performed to compare transient behavior of a regular $\phi_{\mathrm{RF}}$ $=\pi$ bSSFP sequence, a " $1-1$ " $\phi_{\mathrm{RF}}=\pi$ sequence and a "1 -2 1" $\phi_{\mathrm{RF}}=0$ sequence (see Fig. 5). The following parameters were used: $\mathrm{T}_{1} / \mathrm{T}_{2}=1200 / 250 \mathrm{~ms}, \alpha_{\max }=80^{\circ}$, $\mathrm{TR}_{l}=4.0 \mathrm{~ms}, \tau=0.6 \mathrm{~ms}$, and no magnetization preparation. The total TR/TE was 4.0/2.0 $\mathrm{ms}$ for the regular sequence, $\mathrm{TR} / \mathrm{TE}=4.6 / 2.3 \mathrm{~ms}$ for the " $1-1$ " sequence, and $\mathrm{TR} / \mathrm{TE}=5.2 / 2.6 \mathrm{~ms}$ for the " $1-21$ " sequence.

Oscillation levels were quantified as the ratio of absolute finite differences in transient magnetization to the steady-state magnetization at $\Delta \phi=0$. At the water resonance, oscillation level is reduced below a $10 \%$ threshold after 40 TRs for regular bSSFP, 20 TRs for the "1 -1 " sequence, and only 5 TRs for the "1 -21 " sequence. At the bSSFP null, this oscillation level was reached after 176 TRs for regular bSSFP, 165 TRs for the "1 1 " sequence, and 91 TRs for the " $1-21$ " sequence.

\section{Phantom Experiment}

To demonstrate simulated magnetization profiles, bSSFP images of a uniform $\mathrm{MnCl}_{2}$-doped water phantom $\left(\mathrm{T}_{1} /\right.$ $\mathrm{T}_{2}=250 / 50 \mathrm{~ms}$ ) were acquired on a $1.5 \mathrm{~T}$ GE Signa Excite scanner (General Electric Healthcare, Milwaukee, MI) with CV/i gradients (a maximum strength of $40 \mathrm{mT} /$ $\mathrm{m}$ and a maximum slew rate of $150 \mathrm{~T} / \mathrm{m} / \mathrm{s}$ ). An additional linear field gradient was applied in the readout direction to create varying precession frequency. The acquisition parameters were: $\alpha_{\max }=80^{\circ}, \mathrm{TR}_{l}=4.0 \mathrm{~ms}$, $\tau=0.9 \mathrm{~ms}, \pm 62.5 \mathrm{kHz}$ bandwidth, $140 \mathrm{~mm}$ field-of-view (FOV), $0.7 \times 0.7 \times 4 \mathrm{~mm}$ resolution and $96 \times 22$ phase encoding. $\quad \mathrm{TR} / \mathrm{TE}=4.6 / 2.3 \mathrm{~ms}$ and $T_{\text {scan }}$ (scan 
FS-ATR

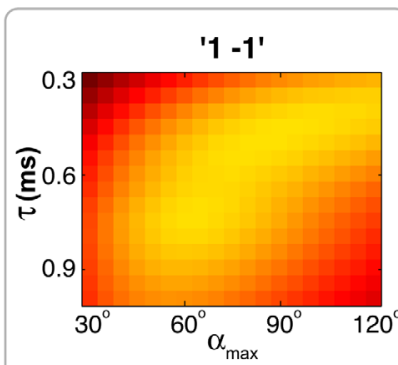

'1 -2 1'

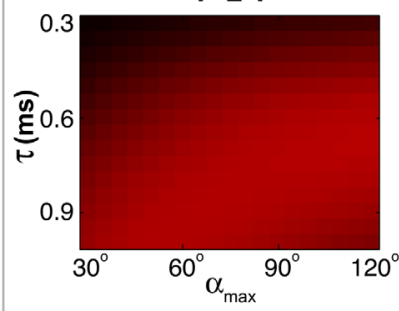

'1 $-33-1$ '

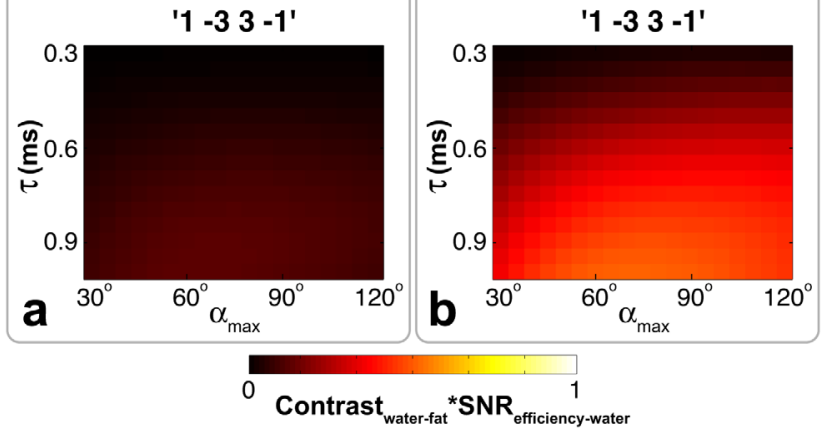

FIG. 3. To find the optimal sequence parameters for water-fat separation at $1.5 \mathrm{~T}$, level of stopband suppression (water-fat contrast) and level of passband signal (water SNR efficiency) were quantified for binomial-pulse bSSFP sequences. An aggregate performance metric was derived as the multiplication of the contrast and SNR efficiency (see color bar). Magnetization profiles were simulated using $T_{1} / T_{2}=1200 / 250 \mathrm{~ms}$ for water, $T_{1} / T_{2}=270 / 85 \mathrm{~ms}$ for fat, and $\mathrm{TR}_{l}=4 \mathrm{~ms}$. a: The aggregate performance metric for the $\phi_{\mathrm{RF}}=\pi$ bSSFP sequence with "1 $1-1$, " "1 -2 1," and "1 -33 -1 " binomial pulses. The "1 -1 " binomial pulse offers better performance compared to higher-order binomial pulses in conventional sequences. b: The aggregate performance metric for the proposed $\phi_{\mathrm{RF}}=0$ bSSFP sequence with " $1-1$, ," "1 -21 ," and "1 -3 $3-1$ " binomial pulses. In general, the proposed sequences outperform the conventional methods for any given order of binomial pulse. Furthermore, the $\phi_{\mathrm{RF}}=0$ sequence with "1 -2 1" achieves the optimal performance among all sequences.

time) $=10 \mathrm{~s}$ with FS-ATR, TR/TE $=5.8 / 2.9 \mathrm{~ms}$ and $T_{\text {scan }}=12 \mathrm{~s}$ with the proposed " $1-21$ ” sequence, and $\mathrm{TR} / \mathrm{TE}=6.7 / 3.4 \mathrm{~ms}$ and $T_{\text {scan }}=14 \mathrm{~s}$ with the " $1-33$ -1 ” sequence.

\section{In Vivo Experiments}

To demonstrate the proposed sequence in vivo, we acquired three-dimensional bSSFP images of the lower leg in healthy subjects. A set of images were collected on a 1.5 T GE Signa scanner equipped with a transmit/ receive extremity coil. The near-optimal parameters found earlier were prescribed for binomial pulses. To prevent bias, the same $\alpha_{\max }$ and $\tau$ values were prescribed for both FS-ATR and the proposed sequence. The acquisition parameters were $\alpha_{\max }=80^{\circ}, \mathrm{TR}_{I}=4.0 \mathrm{~ms}, \tau=0.9$ $\mathrm{ms}, \pm 62.5 \mathrm{kHz}$ bandwidth, $256 \mathrm{~mm}$ FOV, $1 \times 1 \times 1 \mathrm{~mm}$ resolution, and $128 \times 128$ phase encoding. $\mathrm{TR} / \mathrm{TE}=4.9 /$ $2.5 \mathrm{~ms}$ and $T_{\text {scan }}=1 \mathrm{~min} 21 \mathrm{~s}$ with FS-ATR, and TR/ $\mathrm{TE}=5.8 / 2.9 \mathrm{~ms}$ and $T_{\text {scan }}=1 \mathrm{~min} 36 \mathrm{~s}$ with the proposed "1 2 1" sequence.

A separate set of images were collected on a 3 T GE Signa scanner equipped with a transmit/receive quadrature extremity coil and $\mathrm{VH} / \mathrm{i}$ gradients (a maximum strength of $40 \mathrm{mT} / \mathrm{m}$ and a maximum slew rate of $150 \mathrm{~T} /$ $\mathrm{m} / \mathrm{s}$ ). To improve reliability against B0 field inhomogeneities, the total TR for each sequence was set to the minimum possible value constrained by gradient and specific absorption rate limitations. The acquisition parameters

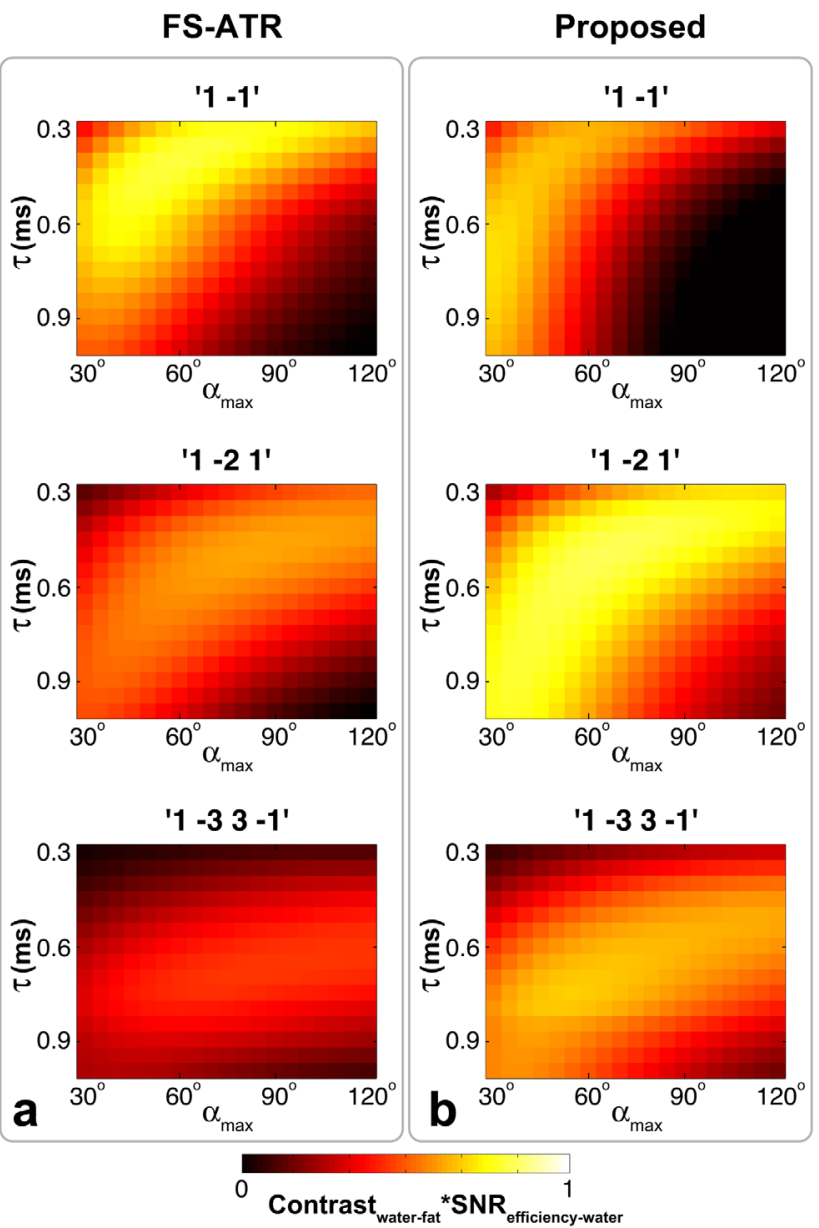

FIG. 4. To find the optimal sequence parameters for water-fat separation at $3 \mathrm{~T}$, level of stopband suppression (water-fat contrast) and level of passband signal (water SNR efficiency) were quantified for binomial-pulse bSSFP sequences. An aggregate performance metric was derived as the multiplication of the contrast and SNR efficiency (see color bar). Simulations were performed using the same set of parameters as in Figure 3. a: The aggregate performance metric for the $\phi_{\mathrm{RF}}=\pi$ bSSFP sequence. The "1-1" binomial pulse offers better performance compared to higherorder binomial pulses in conventional sequences. b: The aggregate performance metric for proposed $\phi_{\mathrm{RF}}=0 \mathrm{bSSFP}$ sequences. The $\phi_{\mathrm{RF}}=0$ sequence with " $1-21$ " achieves the optimal performance among all sequences. 

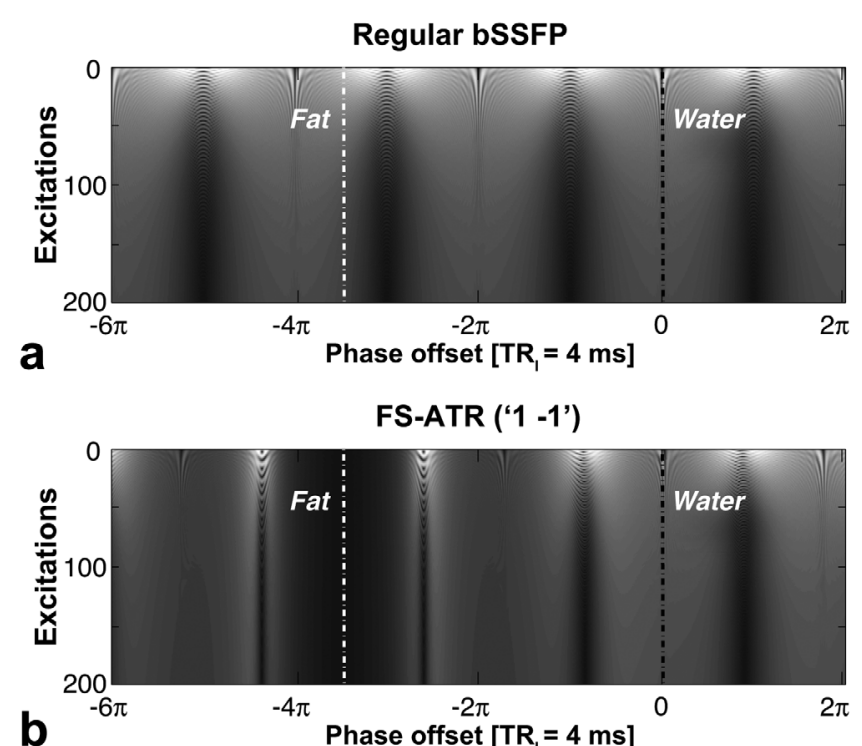

b

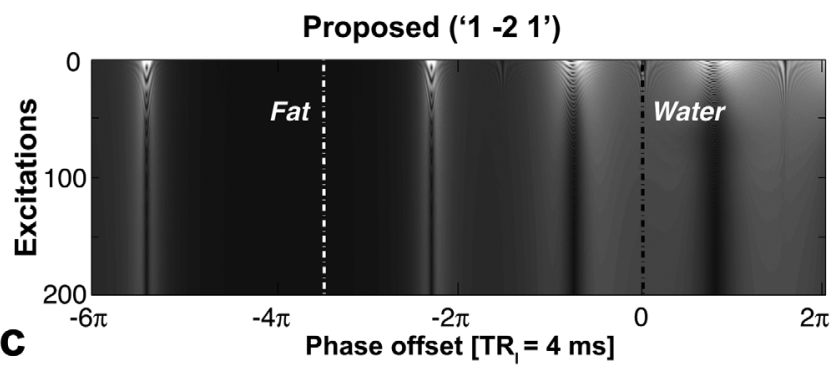

FIG. 5. Transient magnetization profiles of bSSFP sequences designed for water-selective imaging at $3 \mathrm{~T}$. Simulations were performed for $\alpha_{\max }=64^{\circ}, \mathrm{T}_{1} / \mathrm{T}_{2}=1200 / 250 \mathrm{~ms}, \mathrm{TR}_{l}=4.0 \mathrm{~ms}$, and $\tau=0.6 \mathrm{~ms}$. The location of water and fat resonances are marked with dashed black and white lines, respectively. a: Transient profile of a regular $\phi_{\mathrm{RF}}=\pi$ bSSFP sequence as a function of number of RF excitations. b: Transient profile of FS-ATR. c: Transient profile of the proposed "1 -21 " sequence. The transient signal oscillations dampen relatively quickly with the proposed sequence compared to both FS-ATR and the regular bSSFP sequence.

were $\alpha_{\max }=64^{\circ}, \mathrm{TR}_{l}=4.5 \mathrm{~ms}, \tau=0.6 \mathrm{~ms}, \pm 62.5 \mathrm{kHz}$ bandwidth, $256 \mathrm{~mm}$ FOV, $1 \times 1 \times 1 \mathrm{~mm}$ resolution and $128 \times 128$ phase encoding. TR/TE $=5.1 / 2.3 \mathrm{~ms}$, and $T_{\text {scan }}=1 \mathrm{~min} 25 \mathrm{~s}$ with FS-ATR, and TR/TE $=5.7 / 2.6 \mathrm{~ms}$ and $T_{\text {scan }}=1 \min 35 \mathrm{~s}$ with the proposed " $1-2 \quad 1$ " sequence. A $1^{\circ}$ bulk rotation was detected between acquisitions using FS-ATR and the proposed sequence. To account for this rotation, acquired volumes were realigned using a rigid-body transformation and a Lanczos kernel of width three. Experimental protocols were approved by our institutional review board, and written informed consent was obtained from all volunteers.

To evaluate sequence performance, blood-fat contrast, muscle-fat contrast, and blood-muscle CNR were quantified. Measurements were performed in 13 equispaced coronal slices spanning across the lower leg. Within a single slice, blood signal was measured across a regionof-interest (ROI) located in popliteal, peroneal, or posterior tibial arteries (ROI size $19 \pm 13$ pixels, mean \pm SD). Muscle signal was measured across a uniform-intensity ROI neighboring the arteries (203 \pm 108 pixels). Average fat signal was measured across four different ROIs located in superior-left, superior-right, anterior-left, and anterior-right regions of the subcutaneous adipose tissue (112 \pm 73 pixels). Noise was measured across an ROI void of tissue signals ( $583 \pm 290$ pixels). Statistical significance of measurements was assessed using nonparametric Wilcoxon signed-rank tests.

\section{RESULTS}

In this work, we propose a binomial $\phi_{\mathrm{RF}}=0$-cycled bSSFP sequence that increases separation between pass and stopbands by $\pi$ radians compared to conventional binomial bSSFP sequences (e.g., FS-ATR). This increased separation enables the use of higher-order binomial pulses while better preserving the passband signal levels (Fig. 1). Optimum sequence parameters including subpulse spacing $(\tau)$ and maximum flip angle $\left(\alpha_{\max }\right)$ were determined through simulations of water-fat contrast and water SNR efficiency at $1.5 \mathrm{~T}$ and $3 \mathrm{~T}$ (Figs. 3 and 4 ). The proposed "1 -21 " binomial-pulse sequence achieves the maximum performance among all sequences.

To validate the simulated magnetization profiles, we acquired bSSFP images of a phantom with an additional linear field gradient in the readout direction. Figure 6 shows the images acquired with FS-ATR and the proposed "1 -2 1" and " $1-3 \quad 3-1$ " sequences. Inspection of Figure 6 suggests a close match between actual and simulated magnetization profiles (Figs. 1 and 2). The phantom images also reveal the degrading effects of a fourth-order binomial pulse on the water passband adjacent to the stopband. This result indicates that the " $1-2$ 1" binomial pulse should be preferred particularly at lower field strengths, where water and fat resonant frequency differences are smaller.

To assess the performance of the proposed sequence in vivo, three-dimensional bSSFP images of the lower leg were acquired at both $1.5 \mathrm{~T}$ and $3 \mathrm{~T}$. Figures 7 and 8 display representative axial and sagittal cross sections acquired with FS-ATR and the proposed "1 -2 1" sequence. Fat suppression is visibly improved across broad regions in the lower leg with the proposed sequence.

Blood-fat contrast, muscle-fat contrast, and bloodmuscle CNR measurements on the acquired images are listed in Table 1 (see also Methods). The proposed sequence significantly enhances both blood-fat and muscle-fat contrast at $1.5 \mathrm{~T}(P<0.001$, Wilcoxon signedrank test) and at $3 \mathrm{~T}(P<0.004)$. This improvement is at the expense of a slightly reduced blood-muscle CNR at $1.5 \mathrm{~T}(P<0.004)$. In contrast, the increased water-fat resonant frequency difference at $3 \mathrm{~T}$ alleviates passband signal reduction with higher-order binomial pulses. As a result, there are no significant differences in bloodmuscle CNR at $3 \mathrm{~T}(P>0.150)$.

\section{DISCUSSION}

In this work, we demonstrate that a $\phi_{\mathrm{RF}}=0$ sequence offers performance benefits compared to a conventional $\phi_{\mathrm{RF}}=\pi$ sequence for binomial-pulse bSSFP imaging. The proposed sequence enables the use of higher-order binomial pulses for a given passband signal level. Therefore, it enhances the uniformity of stopband suppression 


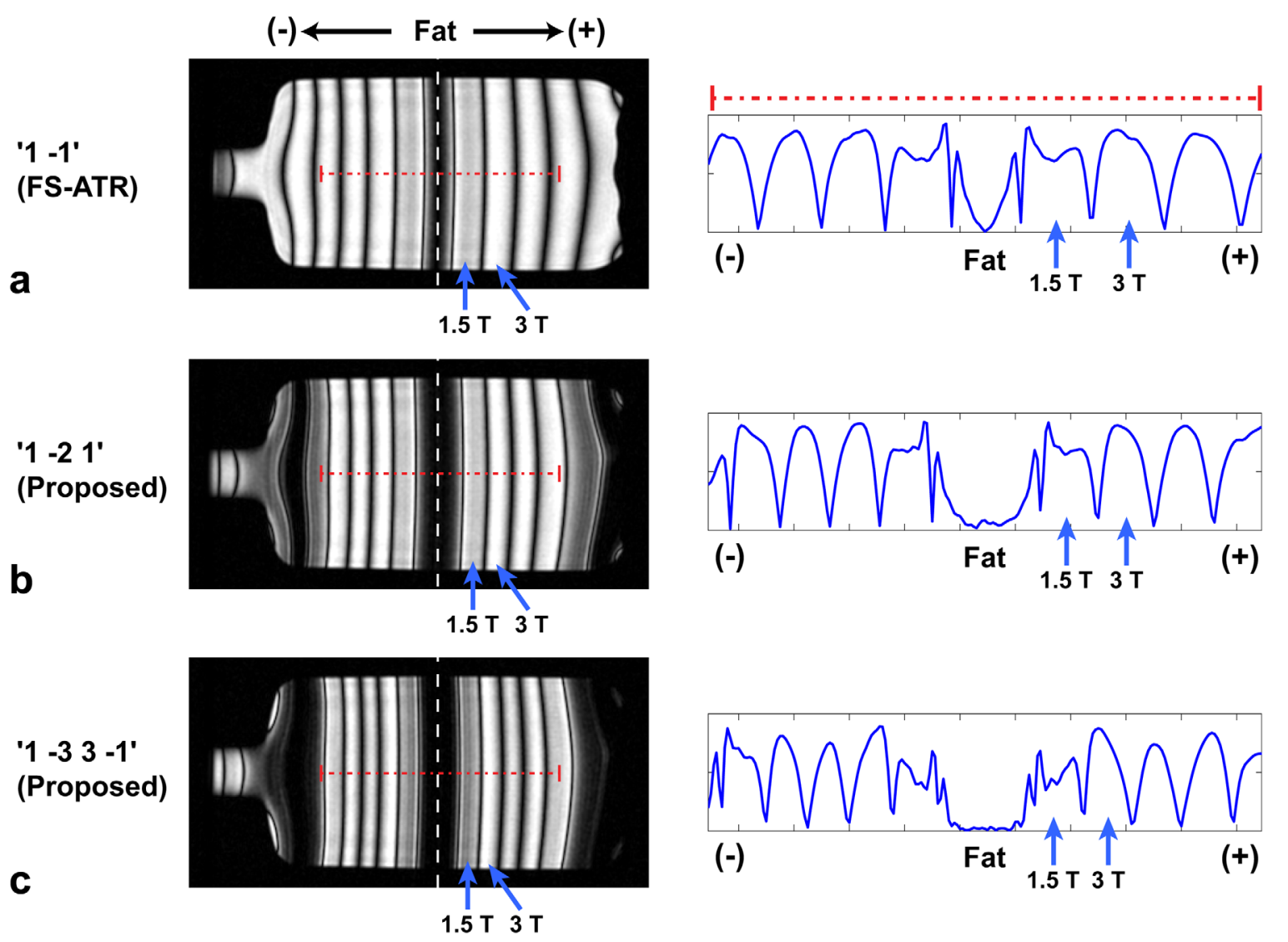

FIG. 6. Balanced SSFP images of a $\mathrm{MnCl}_{2}$-doped water phantom $\left(\mathrm{T}_{1} / \mathrm{T}_{2}=250 / 50 \mathrm{~ms}\right)$ acquired using FS-ATR (a), and a $\phi_{\mathrm{RF}}=0 \mathrm{bSSFP}$ sequence with a "1 -21 " (b), and with a "1 $-33-1$ " binomial pulse (c). TR=4.6, 5.8, and $6.7 \mathrm{~ms}$ for FS-ATR and the proposed " $1-2$ 1 " and "1 $-33-1$ " sequences, respectively. The corresponding magnetization profiles are displayed across a central cross-section of the phantom images (dotted red line segment, right column). A linear field gradient was applied on the horizontal direction to create spatially varying precession frequency in that direction. The center of the stopband (i.e., fat resonance) is annotated with a dashed white line. Centers of the water passbands to be used at $1.5 \mathrm{~T}$ and $3 \mathrm{~T}$ are marked with arrows.

while maintaining high signal efficiency. Furthermore, the proposed sequence aligns the null point of the binomial excitation profile with a bSSFP null as opposed to the center of a passband (Fig. 1). As a result, the proposed sequence generates a stopband that is twice as broad as that of the conventional binomial bSSFP sequence. Taken together, these improvements make the proposed sequence more adept for spectrally selective imaging under moderate to large B0 field inhomogeneity.

Simulated magnetization profiles indicate that the proposed sequence achieves near-optimal performance across a broad range of subpulse spacings and maximum flip angles for the binomial pulse. Meanwhile, a "1 -2 1 " pulse inserted into the $\phi_{\mathrm{RF}}=0$ sequence achieves the maximum water-fat contrast and water SNR efficiency at both $1.5 \mathrm{~T}$ and $3 \mathrm{~T}$. These results suggest that the proposed sequence is considerably robust against B0 and B1 field inhomogeneities, and that a third-order binomial pulse yields the optimum performance. Note, however, that fourth or higher-order pulses may offer improved performance in two-dimensional imaging due to limited B0 field inhomogeneity, and at higher field strengths due to increased separation between water and fat resonances.
The use of higher-order binomial pulses inevitably lengthens the total TR, increasing susceptibility to residual fat signals and banding artifacts in regions of large B0 field inhomogeneity. This may be a particularly limiting factor for field strengths of $7 \mathrm{~T}$ and above. In such cases, water passbands can be broadened by combining multiple acquisitions with the center of the passband shifted to higher and lower frequencies around the water resonance $(38,39)$. Meanwhile, stopband suppression can be maintained by performing a minimum-intensity projection across fat pixels in separate acquisitions, which can be identified through the image phase (29).

The performance metric used here is resilient against global variations in contrast or SNR efficiency, but it is sensitive to the relative normalized ranges (e.g., range/ mean) of these factors. For example, if SNR efficiency is relatively more uniform across the parameter space (e.g., $\left.\tau, \alpha_{\max }\right)$, contrast will be weighted more heavily. As a result, sequence parameters that yield higher contrast will be given preference. Thus the chosen metric inherently emphasizes the factor for which greater improvement can be achieved by tuning of sequence parameters. If strictly balanced weighting is desired, contrast and SNR efficiency values can be normalized prior to the 
'1 - 1 ' (FS-ATR)

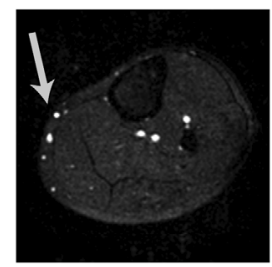

a
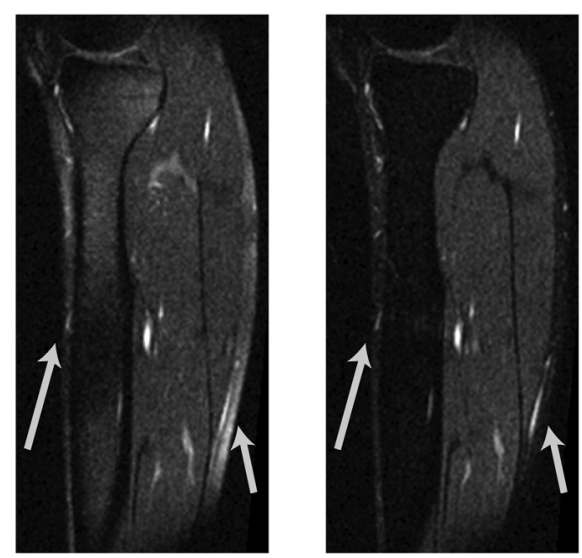

FIG. 7. Balanced SSFP images of the lower leg of a healthy volunteer acquired at $1.5 \mathrm{~T}$ using FS-ATR (left column), and the proposed "1 -2 1" sequence (right column). Axial (a) and sagittal slices (b) are shown with identical display windowing for both sequences. The proposed sequence achieves relatively more uniform fat suppression across broad regions. Arrows point to locations of visible improvement in fat suppression with the proposed technique.

calculation of the metric. In applications with requirements on minimum SNR or contrast levels, the parameter search can be cast as a constrained optimization problem and the metric can be defined as a weighted linear combination of contrast and SNR efficiency (40).

The angiography application considered here uses 3D imaging over large volumes, and it does not require spatially selective excitations. In principle, noncomposite spectrally selective pulses can be used to improve fat suppression for the same pulse duration or to attain the same suppression for a shorter pulse duration. In either case the use of spectral pulses can reduce specific absorption rate deposition compared to binomial pulses. Nonetheless binomial pulses make it easier to adapt the proposed strategy to slab- and slice-selective imaging applications. Furthermore, because spectral selection in binomial pulses is due to free precession during subpulse intervals, these pulses are also more robust against B1 inhomogeneity compared to spectral pulses (37). This benefit can also be attained by other composite pulses based on symmetric filter coefficients such as movingaverage filters. However, we prefer binomial pulses that generate smooth, approximately Gaussian spectral profiles with minimal pass and stopband ripples, minimizing image artifacts $(27,33,34,37)$.

Spectral-shaping based on binomial pulses inherently relies on the assumption that relaxation effects during the subpulse intervals are negligible. While imaging with

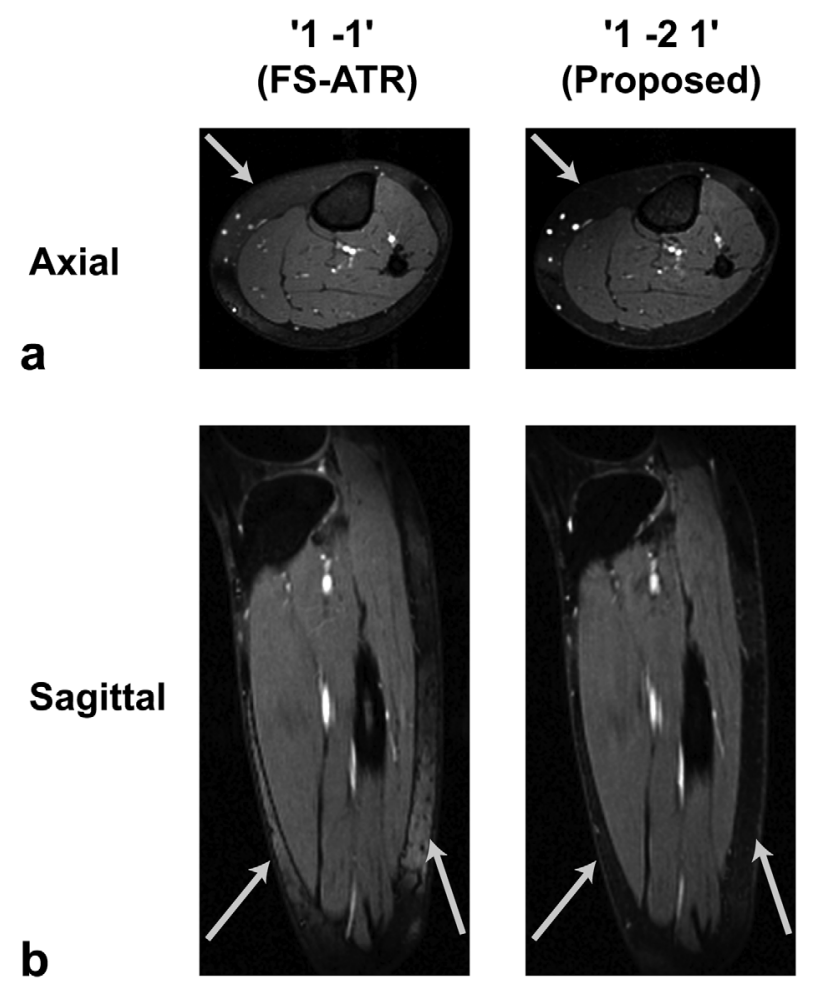

FIG. 8. Balanced SSFP images of the lower leg of a healthy volunteer acquired at 3 T using FS-ATR (left column), and the proposed "1 -2 1" sequence (right column). Axial (a) and sagittal slices (b) are shown with identical display windowing for both sequences. The proposed sequence achieves more reliable fat suppression across the lower leg compared with the conventional technique. Arrows point to locations of improved fat suppression.

relatively longer TRs or targeting short $\mathrm{T}_{2}$ tissues, designs based on this approximation may be suboptimal. In such cases, composite pulses can be used with complex patterns of variable subpulse spacings, flip angles, and phases. Sequence optimization over such large parameter spaces can then be performed by setting up a search algorithm as proposed by Lee et al. (40).

Table 1

In Vivo Contrast Measurements

\begin{tabular}{lccc}
\hline & FS-ATR & Proposed & Significance \\
\hline Measurements at $1.5 \mathrm{~T}$ & & & \\
Contrast $_{\text {blood-fat }}$ & $2.41 \pm 0.24$ & $5.17 \pm 0.57$ & $P<0.001$ \\
Contrast $_{\text {muscle-fat }}$ & $1.07 \pm 0.07$ & $2.44 \pm 0.09$ & $P<0.001$ \\
CNR $_{\text {blood-muscle }}$ & $15.72 \pm 3.01$ & $12.86 \pm 2.42$ & $P<0.004$ \\
$T_{\text {scan }}$ & $1 \mathrm{~min} 21 \mathrm{~s}$ & $1 \mathrm{~min} 36 \mathrm{~s}$ & \\
Measurements at $3 \mathrm{~T}$ & & \\
Contrast $_{\text {blood-fat }}$ & $2.55 \pm 0.25$ & $4.35 \pm 0.58$ & $P<0.004$ \\
Contrast $_{\text {muscle-fat }}$ & $1.83 \pm 0.07$ & $3.41 \pm 0.28$ & $P<0.001$ \\
CNR $_{\text {blood-muscle }}$ & $38.82 \pm 7.92$ & $37.57 \pm 8.08$ & $P>0.150$ \\
$T_{\text {scan }}$ & $1 \mathrm{~min} 25 \mathrm{~s}$ & $1 \mathrm{~min} 35 \mathrm{~s}$ & \\
\hline
\end{tabular}

Blood-fat contrast, muscle-fat contrast and blood-muscle CNR measurements were performed on lower leg images acquired at $1.5 \mathrm{~T}$ and $3 \mathrm{~T}$. Measurements were performed across 13 coronal slices equispaced to span across the entire volume. Mean and standard deviation of the measurements are listed along with the scan times for FS-ATR and the proposed sequence. Significant differences were tested using Wilcoxon signed-rank tests. 
Binomial pulse bSSFP sequences are expected to exhibit similar sensitivity to flow-related ghost artifacts as regular bSSFP sequences (31). Nonzero flow moment of the phase-encode gradients in binomial bSSFP acquisitions, causes some ghosting artifacts near the superior end of lower leg angiograms. Because this study focuses on excitation schemes for spectral-shaping of bSSFP profiles, residual flow artifacts due to spatial encoding do not bias the performance comparisons reported here. Nonetheless, future work will focus on dampening this unwanted flow sensitivity using improved phase-encode gradients with zero first-order moments $(41,42)$.

\section{CONCLUSION}

Spectral-shaping techniques for bSSFP imaging pose a fundamental trade-off between stopband uniformity and passband signal. While stopband suppression can often be improved by more complex patterns of RF excitations and repetition times, this improvement is at the expense of a degraded passband. The proposed binomial wideband bSSFP sequence achieves a more favorable tradeoff compared to conventional binomial bSSFP sequences, by increasing the separation between pass and stopbands. At the same time, it is also more reliable against variations in excitation parameters and Bo field inhomogeneities. Therefore, binomial wideband bSSFP can be a useful technique for spectrally selective imaging when large field inhomogeneities are expected.

\section{APPENDIX}

In this section, we first provide analytical expressions for the sequence- and tissue-parameters dependent terms in the bSSFP signal equation Eq. [1]:

$$
\begin{gathered}
M_{\mathrm{ss}}=i M_{\mathrm{o}} e^{-\frac{\mathrm{TE}}{\mathrm{T}_{2}}} \frac{\left(1-E_{1}\right) \sin \alpha}{1-E_{1} \cos \alpha-\left(E_{1}-\cos \alpha\right) E_{2}^{2}} \\
B=\frac{E_{2}\left(1-E_{1}\right)(1+\cos \alpha)}{1-E_{1} \cos \alpha-\left(E_{1}-\cos \alpha\right) E_{2}^{2}}
\end{gathered}
$$

where $E_{1,2}=e^{-\frac{\mathrm{TR}}{\mathrm{T}_{1,2}}}, \mathrm{TR} / \mathrm{TE}$ are the repetition and echo times, respectively.

Next, we present detailed derivations of the approximate bSSFP signal equations in Eqs. [1] and [4]. The former equation is an approximation to the passband signal for an RF phase increment of $\phi_{\mathrm{RF}}=\pi$ and an offresonant frequency shift of $\Delta \phi=0$. Starting with the complete expression in Eq. [1]:

$$
\begin{gathered}
M_{\mathrm{pass}}(\alpha)=\frac{M_{o}\left(1-E_{1}\right)\left(1+E_{2}\right) \sin \alpha}{1-E_{1} \cos \alpha-\left(E_{1}-\cos \alpha\right) E_{2}^{2}+E_{2}\left(1-E_{1}\right)(1+\cos \alpha)} \\
=\frac{M_{o}\left(1-E_{1}\right)\left(1+E_{2}\right) \sin \alpha}{\left(E_{1}-\cos \alpha\right)\left(1-E_{2}^{2}\right)+\left(1-E_{1}\right)\left(1+E_{2}\right)(1+\cos \alpha)} \\
=\frac{M_{o} \sin \alpha}{\frac{\left(E_{1}-\cos \alpha\right)\left(1-E_{2}\right)}{\left(1-E_{1}\right)}+(1+\cos \alpha)}
\end{gathered}
$$

When TR $\ll\left(T_{1}, T_{2}\right)$, a good approximation to the exponential decay terms is $E_{1,2} \approx\left(1-\frac{\mathrm{TR}}{\mathrm{T}_{1,2}}\right)$. With this substitution, the final expression for the passband signal can be obtained:

$$
\begin{gathered}
M_{\text {pass }}(\alpha) \approx \frac{M_{o} \sin \alpha}{\frac{(1-\cos \alpha)\left(\frac{\mathrm{TR}}{\mathrm{T}_{2}}\right)}{\left(\frac{\mathrm{TR}}{\mathrm{T}_{1}}\right)}+(1+\cos \alpha)} \\
\approx \frac{M_{o} \sin \alpha}{\left(\frac{\mathrm{T}_{1}}{\mathrm{~T}_{2}}+1\right)-\left(\frac{\mathrm{T}_{1}}{\mathrm{~T}_{2}}-1\right) \cos \alpha} .
\end{gathered}
$$

The approximate signal equation for the bSSFP signal null can also be derived from the complete analytical expression for an RF phase increment of $\phi_{\mathrm{RF}}=0$ and an off-resonant frequency shift of $\Delta \phi=0$. Starting again with the complete expression in Eq. [1]:

$$
\begin{gathered}
M_{\mathrm{null}}(\alpha)=\frac{M_{o}\left(1-E_{1}\right)\left(1-E_{2}\right) \sin \alpha}{1-E_{1} \cos \alpha-\left(E_{1}-\cos \alpha\right) E_{2}^{2}-E_{2}\left(1-E_{1}\right)(1+\cos \alpha)} \\
=\frac{M_{o}\left(1-E_{1}\right)\left(1-E_{2}\right) \sin \alpha}{\left(E_{1}-\cos \alpha\right)\left(1-E_{2}^{2}\right)+\left(1-E_{1}\right)\left(1-E_{2}\right)(1+\cos \alpha)} \\
=\frac{M_{o} \sin \alpha}{\frac{\left(E_{1}-\cos \alpha\right)\left(1+E_{2}\right)}{\left(1-E_{1}\right)}+(1+\cos \alpha)}
\end{gathered}
$$

Using the aforementioned first-order approximations for the exponential decay terms under the condition that $\mathrm{TR} \ll\left(\mathrm{T}_{1}, \mathrm{~T}_{2}\right):$

$$
\begin{gathered}
M_{\text {null }}(\alpha) \approx \frac{M_{o} \sin \alpha}{\frac{\left(1-\frac{\mathrm{TR}}{\mathrm{T}_{1}}-\cos \alpha\right)\left(2-\frac{\mathrm{TR}}{\mathrm{T}_{2}}\right)}{\left(\frac{\mathrm{TR}}{\mathrm{T}_{1}}\right)}+(1+\cos \alpha)} \\
\approx \frac{M_{o} \sin \alpha}{\left(\frac{2 \mathrm{~T}_{1}}{\mathrm{TR}}-\frac{\mathrm{T}_{1}}{\mathrm{~T}_{2}}-1+\frac{\mathrm{TR}}{\mathrm{T}_{2}}\right)-\left(\frac{2 \mathrm{~T}_{1}}{\mathrm{TR}}-\frac{\mathrm{T}_{1}}{\mathrm{~T}_{2}}-1\right) \cos \alpha} .
\end{gathered}
$$

\section{ACKNOWLEDGMENT}

The author would like to thank Dwight G. Nishimura and William Overall for helpful discussions on the manuscript.

\section{REFERENCES}

1. Carr HY. Steady-state free precession in nuclear magnetic resonance. Phys Rev 1958;112:1693-1701.

2. Oppelt A, Graumann R, Barfuss H, Fischer H, Hartl W, Shajor W. FISP—a new fast MRI sequence. Electromedica 1986;54:15-18.

3. Scheffler K, Lehnhardt S. Principles and applications of balanced SSFP techniques. Eur Radiol 2003;13:2409-2418.

4. Deshpande VS, Shea SM, Laub G, Simonetti OP, Finn JP, Li D. 3D magnetization-prepared True-FISP: a new technique for imaging coronary arteries. Magn Reson Med 2001;46:494-502.

5. Stuber M, Weiss RG. Coronary magnetic resonance angiography. J Magn Reson Imaging 2007;26:219-234.

6. Maki JH, Wilson GJ, Eubank WB, Glickerman DJ, Millan JA, Hoogeveen RM. Navigator-gated MR angiography of the renal arteries: 
a potential screening tool for renal artery stenosis. AJR Am J Roentgenol 2007;188:540-546.

7. Çukur T, Lee JH, Bangerter NK, Hargreaves BA, Nishimura DG. Noncontrast-enhanced flow-independent peripheral MR angiography with balanced SSFP. Magn Reson Med 2009;61:1533-1539.

8. Gold GE, Hargreaves BA, Reeder SB, et al. Balanced SSFP imaging of the musculoskeletal system. J Magn Reson Imaging 2007;25:270-278.

9. Hargreaves BA, Gold GE, Beaulieu CF, Vasanawala SS, Nishimura DG, Pauly JM. Comparison of new methods for magnetic resonance imaging of articular cartilage. Magn Reson Med 2003;49:700-709.

10. Heyn C, Bowen CV, Rutt BK, Foster PJ. Detection threshold of single SPIO-labeled cells with FIESTA. Magn Reson Med 2005;53:312-320.

11. Çukur T, Yamada M, Overall WR, Yang P, Nishimura DG. Positive contrast with alternating repetition time SSFP (PARTS): a fast imaging technique for SPIO-labeled cells. Magn Reson Med 2010;63:427437.

12. Bieri O, Patil S, Quick HH, Scheffler K. Morphing steady-state free precession. Magn Reson Med 2007;58:1242-1248.

13. Dharmakumar R, Koktzoglou I, Li D. Factors influencing fast low angle positive contrast steady-state free precession (FLAPS) magnetic resonance imaging. Phys Med Biol 2007;52:3261-3272.

14. Scheffler K, Hennig J. T1 quantification with inversion recovery TrueFISP. Magn Reson Med 2001;45:720-723.

15. Schmitt P, Griswold MA, Jakob PM, Kotas M, Gulani V, Flentje M, Haase A. Inversion recovery truefisp: quantification of T1, T2, and spin density. Magn Reson Med 2004;51:661-667.

16. Bieri O, Mamisch TC, Trattnig S, Scheffler K. Optimized balanced steady-state free precession magnetization transfer imaging. Magn Reson Med 2008;60:1261-1266.

17. Hargreaves BA, Vasanawala SS, Nayak KS, Hu BS, Nishimura DG. Fat-suppressed steady-state free precession imaging using phase detection. Magn Reson Med 2003;50:210-213.

18. Vasanawala SS, Pauly JM, Nishimura DG. Linear combination steadystate free precession MRI. Magn Reson Med 2000;43:82-90.

19. Huang TY, Chung HW, Wang FN, Ko CW, Chen CY. Fat and water separation in balanced steady-state free precession using the Dixon method. Magn Reson Med 2004;51:243-247.

20. Reeder SB, Wen Z, Yu H, Pineda AR, Gold GE, Markl M, Pelc NJ. Multicoil Dixon chemical species separation with an iterative least squares estimation method. Magn Reson Med 2004;51:35-45.

21. Çukur T, Nishimura DG. Fat-water separation with alternating repetition time balanced SSFP. Magn Reson Med 2008;60:479-484.

22. Scheffler K, Heid O, Hennig J. Magnetization preparation during the steady-state: fat-saturated 3D true FISP. Magn Reson Med 2001;45: 1075-1080.

23. Derbyshire JA, Herzka DA, McVeigh ER. S5FP: spectrally selective suppression with steady state free precession. Magn Reson Med 2005;54:918-928.

24. Paul D, Hennig J, Zaitsev M. Intrinsic fat suppression in TIDE balanced steady-state free precession imaging. Magn Reson Med 2006; 56:1328-1335.
25. Overall WR, Nishimura DG, Hu BS. Steady-state sequence synthesis and its application to efficient fat-suppressed imaging. Magn Reson Med 2003;50:550-559.

26. Absil J, Denolin V, Metens T. Fat attenuation using a dual steadystate balanced-SSFP sequence with periodically variable flip angles. Magn Reson Med 2006;55:343-351.

27. Kornaat PR, Doornbos J, van der Molen AJ, Kloppenburg M, Nelissen RG, Hogendoorn PCW, Bloem JL. Magnetic resonance imaging of knee cartilage using a water selective balanced steady-state free precession sequence. J Magn Reson Imaging 2004;20:850-856.

28. Leupold J, Hennig J, Scheffler K. Alternating repetition time balanced steady state free precession. Magn Reson Med 2006;55:557-565.

29. Çukur T, Nishimura DG. Multiple repetition time balanced steadystate free precession imaging. Magn Reson Med 2009;62:193-204.

30. Hargreaves BA, Vasanawala SS, Pauly JM, Nishimura DG. Characterization and reduction of the transient response in steady-state MR imaging. Magn Reson Med 2001;46:149-158.

31. Nayak KS, Lee HL, Hargreaves BA, Hu BS. Wideband SSFP: alternating repetition time balanced steady state free precession with increased band spacing. Magn Reson Med 2007;58:931-938.

32. Çukur T, Nishimura DG. Balanced SSFP imaging with variable tip angles and repetition times. In: Proceedings of the 17th Annual Meeting of ISMRM, Honolulu, 2009. p. 2660.

33. Lin HY, Raman SV, Chung YC, Simonetti OP. Rapid phase-modulated water excitation steady-state free precession for fat suppressed cine cardiovascular MR. J Cardiovasc Magn Reson 2008;10:22.

34. Bieri O, Mamisch TC, Trattnig S, Kraff O, Ladd ME, Scheffler K. Optimized spectrally selective steady-state free precession sequences for cartilage imaging at ultra-high fields. MAGMA 2008;21:87-94.

35. Lauzon ML, Frayne R. Analytical characterization of RF phase-cycled balanced steady-state free precession. Concepts Magn Reson 2009; 34A:133-143.

36. Schmitt P, Griswold MA, Gulani V, Haase A, Flentje M, Jakob PM. A simple geometrical description of the TrueFISP ideal transient and steady-state signal. Magn Reson Med 2006;55:177-186.

37. Bernstein MA, King KF, Zhou XJ. Handbook of MRI pulse sequences, 1st ed. Burlington, MA: Elsevier Academic Press; 2004.

38. Bangerter NK, Hargreaves BA, Vasanawala SS, Pauly JM, Gold GE, Nishimura DG. Analysis of multiple-acquisition SSFP. Magn Reson Med 2004:51:1038-1047.

39. Çukur T, Bangerter NK, Nishimura DG. Enhanced spectral shaping in steady-state free precession imaging. Magn Reson Med 2007;58:12161223.

40. Lee KJ, Lee HL, Hennig J, Leupold J. Use of simulated annealing for the design of multiple repetition time balanced steady-state free precession imaging. Magn Reson Med 2012;68:220-226.

41. Markl M, Alley MT, Elkins CJ, Pelc NJ. Flow effects in balanced steady state free precession imaging. Magn Reson Med 2003;50:892-903.

42. Nayak KS, Hargreaves BA, Hu BS, Nishimura DG, Pauly JM, Meyer CH. Spiral balanced SSFP cardiac imaging. Magn Reson Med 2005; 53:1468-1473. 